\title{
The logical consistency of person perception ${ }^{1}$
}

\author{
DAVID A. SUMMERS AND GERALD R. ONCKEN \\ UNIVERSITY OF ILLINOIS
}

Ss made both conditional and unconditional probability estimates concerning persons (Negro and white) and personattributes. When these estimates were evaluated in terms of the axioms of probability theory, it was found that Ss tended toward logical consistency but that consistency varied according to the characteristics of the stimulus person (Negro or white). Furthermore, the differential effects of Negro-white stimulus characteristics upon consistency were greater for white than for Negro Ss.

Following Brunswik's (1956) conception of man as an "intuitive statistician," investigators have shown that Ss can effectively integrate complex information in probabilistic tasks. For example, recent studies (e.g., Peterson \& Miller, 1965) have reported that Ss are near optimal in their estimates of the probability with which an observable datum (e.g.. either a black or a white marble) is associated with an unobservable data generator (e.g., an urn containing either predominantly black or predominantly white marbles). Moreover, when Ss make both conditional and unconditional probability estimates in this type of task, they generally achieve a high degree of internal consistency in their judgments (Peterson, Ulehla, Miller, Bourne, \& Stilson, 1965). Here, the criterion for consistency is given by the definition of conditional probability:

$\mathrm{P}(\mathrm{A}) \mathrm{P}(\mathrm{B} / \mathrm{A})=\mathrm{P}(\mathrm{B}) \mathrm{P}(\mathrm{A} / \mathrm{B})$

where $A$ and $B$ refer to the datum and data generator, respectively.

Note, however, that efforts to study the internal consistency of subjective probabilities have typically been limited to "neutral" tasks (e.g., marbles and urns). As a result, little attention has been given to logical consistency in a domain in which Ss frequently make probabilistic judgments-namely, person perception. Although probabilistic judgments regarding person attributes have been utilized to construct models of cognitive structure (Todd \& Rappoport, 1964), it remains to be shown whether Ss are logically consistent in this domain.

The present study extends the model described above to probabilistic judgments concerning persons and person attributes as follows:

$$
\mathrm{P}(\mathrm{SP}) \mathrm{P}(\mathrm{A} / \mathrm{SP})=\mathrm{P}(\mathrm{A}) \mathrm{P}(\mathrm{SP} / \mathrm{A})
$$

where SP and A refer to Stimulus Persons (e.g., American, Frenchman, Negro, white, etc.) and person attributes (e.g., honest, industrious, etc.), respectively, and where consistency is achieved to the extent that the two sides of the equation covary over a domain of persons and/or attributes.

The experiment reported here concerns the logical consistency with which Ss make probabilistic judgments about persons and person attributes, and focuses on the extent to which consistency varies as a function of (a) race of the stimulus person, (b) race of the $S$, and (c) semantic characteristics of the attributes in question.

\section{Method and Procedure}

Design. Twenty Negro and 30 white male undergraduates made judgments about two types of stimulus persons (Negro vs white) over 24 attributes from three semantic domains. The experiment thus takes the form of a 2 (Subjects) by 2 (Stimulus Persons) by 3 (Attributes) factorial design.

Task Judgments. All Ss made unconditional estimates regarding the occurrence of (a) person attributes and (b) Negroes and whites in the population. In addition, Ss made conditional estimates regarding all possible attribute-SP (i.e, Negro or white) pairs.

Unconditional estimates were obtained as follows: "Given 100 persons, how many would you expect to be " where estimates were obtained for each of the 24 attributes, as well as for "Negro" and "white."

For SP-given-attribute estimates, items assumed the form of the following example: "Given $100 \mathrm{moral}$ persons, how many would you expect to be Negro?" For the attribute-given-SP estimates, the form was reversed: "Given 100 Negroes, how many would you expect to be moral?"

The 24 attributes represented three factors (eight attributes each) derived from pooled Negro-white responses to a semantic differential: Evaluative (e.g., moral, immoral); Excitability (e.g., excitable, calm); and Typicality (e.g., typical, unique).

All items were accompanied by a 100-point response scale. The items were assembled in booklets, where the order of the items was appropriately counterbalanced with respect to Negro vs white stimuli. Results and Discussion

The internal consistency of each S's subjective probabilities was evaluated in terms of Equation (2). Specifically, Ss' responses yielded 48 pairs of subjective probability products. Consistency was measured by the correlation between these products, i.e., between the two sides of Equation (2). This procedure produced six consistency correlations (three attribute domains by two stimulus persons) for each $\mathrm{S}$.

Consistency correlations were transformed into Fisher's Z coefficients, and analyzed according to a 2 (Subjects) by 2 (Stimuli) by 3 (Attributes) analysis of variance with repeated measures over the last two factors. This analysis revealed that consistency varied as a function of Stimuli ( $F=21.59, \mathrm{df}=1 / 48, \mathrm{p}<.001$ ). Specifically, internal consistency was greater for white stimulus persons than for Negro stimulus persons (see Table 1). 
Table 1

Mean Consistency Correlations According to Subjects, Stimuli and Attributes

\begin{tabular}{llccc} 
& & \multicolumn{2}{c}{ Subjects } & \\
\cline { 3 - 4 } Stimuli & Attributes & Negro & White & Row Means \\
\hline \multirow{4}{*}{ Negro } & Evaluative & .69 & .71 & .70 \\
& Excitable & .57 & .65 & .61 \\
& Typicality & .68 & .72 & .70 \\
& (Mean) & $(.65)$ & $(.69)$ & $(.67)$ \\
\hline \multirow{4}{*}{ White } & Evaluative & .74 & .90 & .83 \\
& Excitable & .76 & .72 & .74 \\
& Typicality & .66 & .88 & .79 \\
& (Mean) & $(.72)$ & $(.83)$ & $(.79)$ \\
\hline \multirow{2}{*}{ Column } & & & & \\
\hline
\end{tabular}

In addition, significant interactions were found for Subjects by Stimuli $(F=4.94, \mathrm{df}=1 / 48, p<.05$ ) and for Subjects by Stimuli by Attributes $(F=4.70, \mathrm{df}=1 / 96$, $p<.025)$. The Subjects by Stimuli interaction can be accounted for by the differential response by Negro and white Ss to the Negro and white stimuli. Thus, while judgments were more consistent for white than Negro stimuli over all conditions, this difference was substantially greater for white Ss $(d=.14)$ than for Negro Ss $(d=.07)$.

With regard to the three-way interaction, the data indicate that the Subjects by Stimuli interaction does not hold for all attribute domains; i.e., for attributes in the Excitability domain, Negro and white Ss were equally consistent when judging both Negro and white stimuli.

These results indicate that (a) Ss tend toward logical consistency in their estimates regarding persons and person attributes, but that (b) consistency is affected by characteristics of both the stimulus and the respondent.

With regard to (a), the consistency correlations obtained here are generally high. It should be pointed out, however, that the coefficients obtained here were substantially lower than those obtained by Peterson et al (1965) in tasks involving neutral stimuli.

Why S's judgments should be less consistent here than in the neutral tasks mentioned above is not entirely clear. One explanation is that responses to the present task were the result of previously learned, or assumed, associations of persons and attributes, and that these associations were often expressed without regard for other, related judgments regarding the SP-attribute pair in question. In short, certain of the Ss' cognitive elements in the domain of person perception are not fully integrated, but are compartmentalized.
Such an explanation is particularly helpful with regard to the finding (b) that consistency is significantly lower for Negro than for white stimulus persons. In this respect, it should be noted that most stereotypic beliefs take the form of "attribute-given Stimulus Person" estimates; e.g., given that SP is a Negro, the probability is high (or low) that he is immoral (or moral). Thus, while $\mathrm{S}$ may have been exposed to stereotypic beliefs of this nature, it is less likely that he will have been exposed to other, logically related beliefs regarding Negroes and morality. As a result, S's conditional and unconditional estimates -at least concerning Negroes and the attribute "morality"-are not fully interrelated in a consistent manner.

Although the relatively low consistency indices evidenced by white Ss for Negro stimuli can be accounted for by stereotypic beliefs, why should Negro Ss have equally low indices for the Negro stimuli? Here, several investigators (e.g., Pettigrew, 1964) report a strong tendency by Negroes to adopt stereotypic beliefs expressed by the white majority. If such is indeed the case, we would expect Negroes (as well as whites) to tend toward inconsistency when making judgments about Negro stimuli.

That the differential effects of Negro vs white stimuli upon consistency were more pronounced for the white than for the Negro Ss suggests that stereotypic beliefs were particularly salient for the white respondents when making estimates about the Negro stimuli.

In conclusion, Ss tend toward logical consistency in their estimates regarding persons and person attributes, but are less than optimal. Moreover, these results suggest that inconsistency can be partially accounted for by stereotypic beliefs about the stimulus persons.

\section{References}

BRUNSWIK, E. Perception and the representative design of psychological experiments. Berkeley: University of California Press, 1956. PETERSON, C., \& MILLER, A. Sensitivity of subjective probability revision. J. exp. Psychol, 1965, 70, 117-121.

PETERSON, C., ULEHLA, Z., MILLER, A., BOURNE, L., \& STILSON, D. Internal consistency of subjective probabilities. J. exp. Psychol., $1965,70,526-533$.

PETTIGREW, T. A. profile of the American Negro. Princeton: Van Nostrand, 1964.

TODD, F., \& RAPPOPORT, L. A cognitive structure approach to person perception: A comparison of two models. J. abnorm. soc. Psychol, 1964, 68, 469-478.

Note

1. This research was supported by ARPA Order No. 454, Contract Nr 177-472, Nonr 1834(36) with the Advanced Research Projects Agency and the Office of Naval Research. The authors thank Frank Taylor for assistance in data collection. 